\title{
What is nanotechnology?
}

\author{
Jeremy J. Ramsden
}

Department of Advanced Materials, Cranfield University, Bedfordshire, UK

The term nanotechnology was first used in 1974 by the late Norio Taniguchi ${ }^{1}$ (University of Tokyo) to refer to the ability to engineer materials precisely at the scale of nanometres. ${ }^{2}$ This is in fact its current meaning; 'engineer materials' is usually taken to comprise the design, characterization, production and application of materials, and the scope has nowadays been widened to include devices and systems rather than just materials. Nanotechnology is thus defined as the design and fabrication of materials, devices and systems with control at nanometre dimensions.

The essence of nanotechnology is therefore size and control. Because of the diversity of applications, the plural term 'nanotechnologies' is preferred by some; nevertheless, they all share the common feature of control at the nanometre scale.

\section{What is nanoscience?}

Sometimes a distinction is made between nanotechnology and nanoscience, the latter focusing on the observation and study of phenomena at the nanometre scale, and ways of manipulating matter at that scale, at which many properties of matter differ from those familiar at larger scales. The distinction is not of great importance, however: the nanotechnologist will perforce have to observe, study and manipulate matter in the course of his or her work. 'Nanoscience' suggests a solid body of theory, upon which a technology could be built; such theory is still inchoate, however, and the nanotechnologist is as likely to contribute to it as the nanoscientist. In this essay the term 'nanotechnology' will be used in an all-embracing sense.

\footnotetext{
${ }^{1}$ N. Taniguchi, “On the basic concept of nano-technology”. Proc. Intl Conf. Prod. Engng Tokyo, Part II (Jap. Soc. Precision Engng).

${ }^{2}$ The nanometre, $10^{-9} \mathrm{~m}$, i.e. one millionth of a millimetre, is the characteristic scale of molecules, i.e. groups of atoms bound covalently together. Atoms are of the order of $1 \AA \AA$ in size, i.e. $0.1 \mathrm{~nm}$; hence a true nano-object would have to contain tens or hundreds of atoms to achieve the requisite size. To get a feel for the smallness of the nanometre, note that within one second human hair grows a few nm, and continents drift by a similar amount.
} 


\section{What is nanobiotechnology?}

Nanobiotechnology and bionanotechnology—-they are essentially synonyms - refer to materials and processes at the nanometre scale that are based on biological, biomimetic or biologically-inspired molecules, and nanotechnological devices used to monitor or control biological processes, e.g. in medicine. An example of the former is the optically switched optical switch incorporating the biomolecule bacteriorhodop $\sin ^{3}$ and an example of the latter is the biochip - an array of known DNA fragments used to capture unknown DNA from a sample.

\section{The relation of nanotechnology to chemistry, biology and quantum mechanics}

Nanotechnology is (rightly) considered to be rather new, but it is by no means the only field concerned with atoms and molecules. In different ways, the disciplines of physics, chemistry and biology have long dealt with atoms and molecules, their behaviour and their manipulation; and quantum mechanics is already firmly established as the science of the absolutely small. What is then really new in nanotechnology?

\section{Chemistry}

Chemistry is a powerful contender for the nanotechnology label. Nevertheless, there is an essential difference in approach. While chemistry also deals with the manipulation of molecules, and hence is no stranger to nanometre dimensions, the chemist does not control systems in the way that the engineer does. Molecules mostly reside in their free energy minima and it requires ingenuity, intuition and luck to steer their precursors along the paths to the desired end product. Chemistry thus lacks the dictatorial element of control over matter as practised by the engineer.

That is not to say that chemistry is not tremendously important to nanotechnology, but until now that importance has been rather negative, in the sense that chemical reality often thwarts the nanotechnologist's desire. A good illustration of this is Eigler and Schweizer's now famous experiment in which they manipulated molecules of xenon on a surface to form the letters 'IBM'. They attempted to generate structures in dictatorial engineering mode, but were ultimately limited by chemistry: they could not put the atoms wherever they wanted, but only at certain lattice sites of the underlying surface, even at the ultralow temperature $(4 \mathrm{~K}$, i.e. just four degrees centigrade above absolute zero) at which they had to work. At present it looks as though chemistry may impose fundamental limitations on the freedom of the nanotechnologist to manipulate matter at the atomic and molecular scales.

\footnotetext{
${ }^{3}$ P. Ormos et al., "Protein-based integrated optical switching and modulation". Appl. Phys. Lett. 80 (2002) 4060-4062.

${ }^{4}$ D.M. Eigler and E.K. Schweizer, "Positioning single atoms with a scanning tunnelling microscope". Nature (Lond.) 344 (1990) 524-526.
} 


\section{Biology}

Biology is considered to provide living proof of principle of nanotechnology. Biological structures at macromolecular and supramolecular scales are apparently assembled using the principles of self-assembly so eagerly sought by the nanotechnologist, and these structures, mostly protein-based, often combine extraordinary lightness with extraordinary strength, and may be miniature mechanisms of marvellous complexity. Two examples of amazing mechanical devices are the so-called $F_{1}$ ATPase enzyme that uses a proton gradient across the membrane in which it is embedded to synthesize that universal biological energy provider, adenosine triphosphate (ATP); and the so-called type III secretion system (TTSS) - a spherical assembly of needles found on the surface of certain pathogenic bacteria, and used to inject poison into their targets.

\section{Quantum mechanics}

Size is mostly a relative term, but quantum mechanics offers a definition of absolute smallness: a system is absolutely small if it is perturbed by the act of observing it. ${ }^{5}$ Thus a photon is usually destroyed by the act of observation, or its state is irretrievably altered. Most nanosystems are not small enough for this to be the case. Nevertheless, quantum effects are needed to understand certain nano-objects, for example the small clusters of atoms called quantum dots, nanodots or nanoparticles. These objects are tiny spheres of a solid, typically a semiconductor. In condensed matter, electrons are no longer the point particles they are believed to be in free space, but have extension, quantified by their Bohr radius, which can vary from a few to hundreds of nanometres, depending on the material. It is possible to make nanoparticles smaller then the Bohr radius of electrons in them, thus the electrons are subjected to quantum confinement, with the observable effect that the optical absorption and fluorescent emission of the particle are shifted towards higher energies, the magnitude of the shift depending on the particle size. Quantum effects must also be considered with ultraminiaturized electronic circuitry—single electron devices.

In summary, physics, chemistry and biology strongly overlap with nanoscience (defined as the study of matter at the molecular scale), but differ essentially from nanotechnology, which seeks to impose control over materials and devices at that scale. Quantum mechanics affects the performance of devices at the lower end of the size range of nano-objects. Nanoscience and nanotechnology could therefore be defined via the convergence of chemistry, biology, physics and engineering. If one emphasizes this unity, it makes sense to refer to nanotechnology in the singular; if one wishes to emphasize the diversity of applications, then it makes sense to refer to nanotechnologies in the plural. ${ }^{6}$

\footnotetext{
${ }^{5}$ There are also "macroscopic" examples of this: asking a question of a person alters her mental state and their answer reflects that altered state, not the state immediately prior to asking the question.

${ }^{6}$ Nanotechnology is sometimes considered as just one of four converging technologies, the other three being biotechnology and bio-medicine, information technology, and cognitive science (the NBICNano-Bio-Info-Cogno - quartet); alternatively all may be considered as part of nanotechnology.
} 


\section{What are the key elements of the technology?}

The technology of realization can be conveniently divided into fabrication (of materials and devices) and metrology. Both these areas are well covered by other essays in this issue, so here we shall only say a few words about them. Fabrication is divided into 'top down' and 'bottom-up' techniques. The former constitutes a seamless continuum of processes becoming ever more miniature (Figure 1). There is now considerable overlap between ever more precise cutting and grinding tools (as epitomized by the Cranfield "Tetraform") and the techniques now dominating the integrated circuit industry, in which patterns are transferred (using photolithography or electron beam lithography) onto semiconductors and material is removed by chemical or ion beam etching.

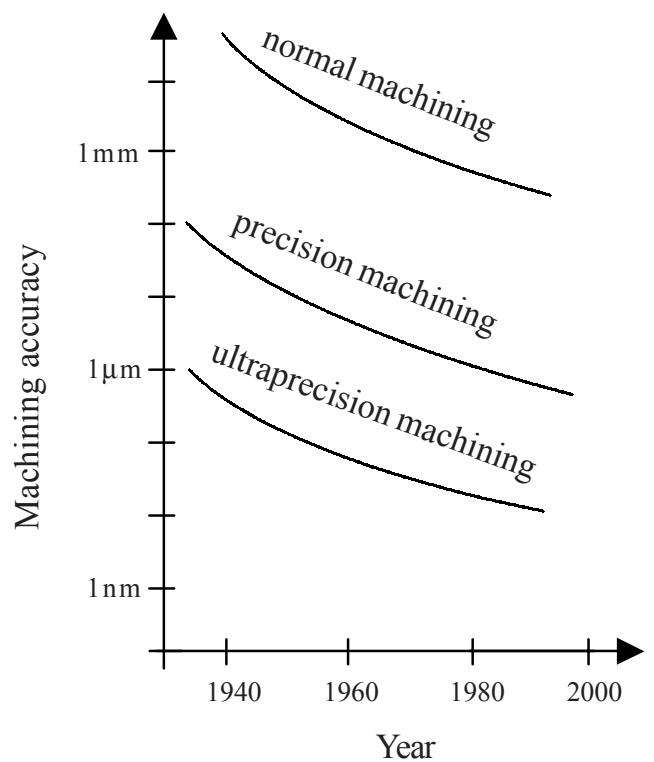

Figure 1. Top down nanotechnology - the evolution of machining accuracy (after N. Taniguchi, "Current status in, future trends of, ultraprecision machining and ultrafine materials processing". Annals CIRP 32 (1983) 573-582).

The photolithography family of techniques is hugely expensive, and hence only economical if vast numbers of replicas can be produced. The large capital expense of these fabrication facilities has driven the search for other methods of making ultrasmall devices, namely by the process called self-assembly or autofabrication. This comprises the production of precursors, typically molecules or simply shaped objects that can be produced en masse at low cost, designed such that when they are mixed togther in a supporting medium, which might be water or an organic liquid, they connect together in a strictly predefined fashion in order to create complex three dimensional structures. The process is therefore more accurately called selfconnecting. An important inspiration for the feasibility of self-connecting fabrication has been the 
assembly of bacteriophage from precursor structures. ${ }^{7}$ No devices of practical, commercial utility are currently made by this route, however. A major difficulty is the current lack of design tools with which manufacturable precursors can be specified for a given final device; most of the work reported in the field describes structures that were observed to be formed from some interesting precursors. Nevertheless, the much lower capital costs of this fabrication route, and hence the possibility of manufacturing smaller quantities of devices, continues to motivate research work in the field.

Self-connecting processes were studied both experimentally and theoretically long before the appearance of nanotechnology. Although they are considered now to belong to it, as are the 'top down' processes which, since Taniguchi's article, have reached the nanometre scale, the most characteristic nanotechnological fabrication process is molecular manufacturing, in which devices are assembled molecule by molecule, or even atom by atom. ${ }^{8}$ The assembly would be carried out by purpose-built assemblers, which would themselves be the final products of a chain of machine tool manufacture, in which machines at one level would make the parts for a smaller machine at the subsequent level, as was suggested by R.P. Feynman in his famous 1959 lecture "There's plenty of room at the bottom". Since the assemblers would themselves be very small, they must also be very numerous if they are to be practically useful, hence they should be able to manufacture copies of themselves (lest this self-replicating capability overtaxes their information storage capacity (since they must in addition build other objects), an alternative would be to reserve the self-replicating ability for the next level up). Although the assembler also has its biological counterparts - subcellular organelles such as the ribosome or the proteasome are considered to be living proofs of principle - nothing remotely resembling an assembler has presently been built, and details of the realization of self-replication are not the principle preoccupation of most nanotechnologists.

\section{Metrology}

Metrology is intrinsic to manufacturing. Any serious manufacturing process must incorporate the means to quantitatively assess the quality of the manufactured objects. This topic is covered in depth elsewhere in this issue; for now it will suffice to mention one of the main tools of the nanometrologist, the atomic force microscope (AFM), now one of the family of scanning probe microscopes. The two essential components of an AFM are a needle capable of responding sensitively to small declivities of a surface, and the means to scan that needle across the surface. In typical realizations of the AFM, the needle is mounted on a flexible, highly reflecting cantilever onto which a collimated beam of light is directed; if for example a small bump is encountered, the needle will be pushed up, moving the cantilever and displacing the beam reflected from it. Scanning is accomplished by applying minute voltages to a piezoelectric

\footnotetext{
${ }^{7}$ E. Kellenberger, “Assembly in biological systems”. In: Polymerization in Biological Systems, CIBA Foundation Symposium 7 (new series). Amsterdam: Elsevier (1972).

${ }^{8}$ K.E. Drexler, Engines of Creation. New York: Doubleday (1986).
} 
crystal on which the sample is mounted, which converts electricity into spatial displacement.

As well as being a tool for metrology, the AFM is also considered to be a kind of prototype assembler, with which atoms can be pushed into place, as in Eigler and Schweizer's experiment already referred to. ${ }^{4}$

\section{What can nanotechnology do for us?}

Here we approach the question of why nanotechnology is often stated to be revolutionary. Let us consider three distinct aspects (Figure 2): indirect, direct and conceptual. By indirect is meant the progressive miniaturization of existing technologies, which opens up new areas of application for those technologies. Direct refers to the application of novel, nanoengineered artifacts, either to enhance the performance of existing processes and materials, or for wholly novel purposes. Finally, there is the conceptual aspect of nanotechnology, in which all materials and processes are considered from a molecular or even atomic viewpoint, as in living systems, in which complicated molecules (like proteins) are broken down into their constituent amino acids, which are then used for the templated synthesis of new proteins. The artificial counterpart of this process is largely untouched territory. Entirely novel integrated manufacturing life cycles await development, in which extreme energy economy and the absence of unpleasant waste products will be prominent. Furthermore, the conceptual nano-viewpoint offers the possibility of a new understanding of the world, its structures and its processes.

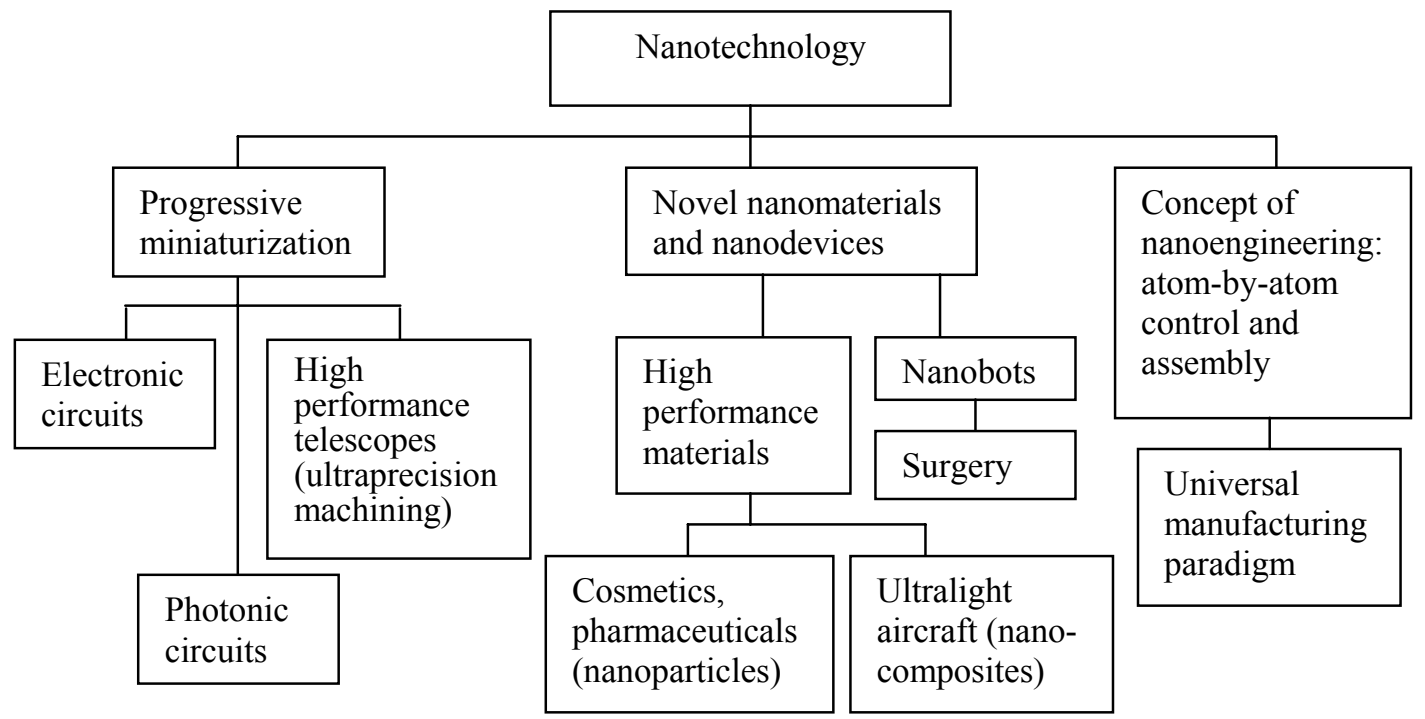

Figure 2. From left to right, the indirect, direct and conceptual branches of nanotechnology, with examples.

Indirect nanotechnology is enabling technology. Miniaturization can quantitatively enhance performance, and when a quantitative change is big enough, it becomes qualitative. A good 
example is provided by the history of the cellular telephone. Based on thermionic valves (vacuum tubes), the circuitry for a cellular telephone would take up the volume of a large multistorey building. The cellphone concept — which dates from the 1950s — only became useful once the circuits and their components became small enough to fit into a handset. The minute size of integrated circuit components enables circuits of far greater power and complexity to be realized than would otherwise be practically possible. All the applications of powerful computing, including the world wide web, are thus epiphenomena of nanotechnology.

Novel forms of matter, such as nanoparticles, or the still-to-be-realized nanosized robots (nanobots), represent direct nanotechnology. There are many advances in this realm whose field of application is such that the nanocomponent is hidden. A good example is nanofoils made from thousands of alternating nanometre-thick layers of two different metals. A brief electrical pulse applied across the foil initiates mixing of the two metals and the release of a large amount of heat, sufficient for highly localized interfacial bonding of the materials between which the nanofoil is placed. Therefore any assemblage whose components are bonded together using this technique is a manifestation of nanotechnology. The assemblage could be very large, such as an aeroplane.

Finally, by conceptual nanotechnology we mean that nanotechnology represents a novel viewpoint from which to survey the world: one in which structures are scrutinized at the nanometre scale, and processes are analysed by considering the movements of each individual atom. This should lead to wholly new ways to understand the world - with the hope that the underlying mechanisms of many hitherto puzzling phenomena will be thereby revealed.

But is this really so new? The answer is that although man often stumbled upon nanomaterials with unique properties - mediaeval stained glass is an example - this was apparently done without being aware of the nanostructuring. By way of illustration, consider that although the properties of steel are now known to be due to structures existing down to the nanometre level, steel was discovered and manufactured largely in ignorance of these structures, and it makes little sense to call steel pioneers such as Bessemer nanotechnologists, any more than it makes sense to call Neanderthal man an early nanotechnologist just because he doubtless produced carbon nanotubes in abundance in his primitive cave fires. Using nanometrology, these nanotubes are now characterized at unprecedented levels of detail, accompanied by insights from computations.

Indeed, the whole realm of computational chemistry and materials science has acquired a mantle of vastly greater significance through nanotechnology than it had previously. Before the nano era, the difficulties faced by the computational scientists were well caricatured by the worker who spent six months computing the density of water from first principles, finally producing the result of $1 \mathrm{~g} / \mathrm{cm}^{3}$, with an uncertainty of, say, $\pm 0.5 \mathrm{~g} / \mathrm{cm}^{3}$, whereas a result with four or more orders more of precision could have been obtained within a couple of minutes in a modern analytical laboratory by simple weighing. The cardinal rule of computational science, that calculations should only be undertaken if the desired result would thereby be obtained more quickly than by experiment, has been broken so often that the discipline became marginalized, 
producing results that were at best confirmatory. Thanks however to the small size of nanoobjects, and to the vastly increased computational power enabled by circuit miniaturization, their properties can now often be explicitly and usefully calculated from first principles, more rapidly than they can be elucidated from experiment.

\section{What has nanotechnology achieved so far?}

The main areas of application to date are in electronics, photonics, pharmaceuticals and cosmetics, and finishes for surfaces and textiles.

In the indirect realm, the most widespread application (of 'top down' nanostructuring of semiconductors) is in circuit miniaturization, leading to exponentially increasing computational power. Hence every manifestation of the expanding rôle of computers, from the world wide web to cellular telephones, is an indirect product of nanotechnology. Closely related to the creation of circuitry is the fabrication of micro electro-mechanical systems (MEMS), in which three-dimensional mechanical structures functioning as accelerometers, torque sensors, pressure sensors and so on, are created in silicon.

In photonics, integrated optics, in which light is confined and modulated in structures smaller than the wavelength of light, i.e. with length scales of the order of $100 \mathrm{~nm}$, is now considered to be a part of nanotechnology. Optical fibres are already very widely used for the transmission of information (telecommunications) over long distances at very high data transmission rates and capacities, and while switching and routing of data packets currently uses hybrid optoelectronic or optoelectromechanical technology, prototype all-optical switches have already been demonstrated. The successful realization of such devices is an essential precursor to the optical computer. The second great area of exploitation of photonics is the realm of sensors, for both physical and chemical parameters. Here, integrated optics offers scope for new types of sensors far surpassing MEMS and electrochemical devices in precision, accuracy and robustness. These photonic devices are presently manufactured by top down technology.

In the direct realm, nanoparticles are already widely used in skin cosmetic formulations. Active ingredients in the form of nanoparticles retain the functionality of microparticles (e.g. the ability to adsorb ultraviolet light), but do not scatter light, hence can be applied without influencing the appearance of the skin. The drawback of minuteness is that the particles can penetrate through the skin into the body almost without let or hindrance, with effects as yet largely unknown.

Nanostructured "superhydrophobic" surfaces, directly inspired by nanoscopic studies of the surfaces of "self-cleaning" leaves such as those of the lotus, can be applied to textiles, window glass etc. with similar effect. Merely rinsing with water scavenges dust and dirt particles from the surface, without, as yet, perceptible effects even among the ranks of cravat and window cleaners. This niche application may however prolong the popularity of the glass cube or parallelepiped among contemporary architects: hence indirectly, nanotechnology even affects the built environment at large scales. 
By incorporating nanoparticles into a synthetic polymer matrix, either some existing attribute of the polymer can be enhanced - a good example is diminished permeability for gases - or some wholly new property, that of the particles, can be acquired by the blend - for example magnetism, by the use of magnetic nanoparticles. The best nanoparticles for enhancing barrier properties (diminishing gas permeability) are flat tablets - like many natural clays — whose presence in the matrix enormously increases the tortuosity of the average path followed by a gas molecule diffusing through the material. These nanocomposites are used extensively in the beverage industry as a thin coating on plastic bottles, which can therefore be made thinner while keeping the same barrier function, or a stronger but more permeable polymer can be used. Enhanced scratch resistance can be conferred by adding nanoparticles of a very hard substance to the polymer, and so on. Nature abounds in such composites, wood being a well-known example, in which cellulose, conferring mechanical strength, is blended with lignin, which glues the cellulose fibres together. The first artificial nanocomposites, based on clays and polyamides and made by dispersing preformed nanoparticles in monomer and polymerizing the matrix around the nanoparticles, were introduced in the early 1990s by Toyota. Other routes to making nanocomposites are blending preformed nanoparticles with the matrix, typically in a molten state; and synthesizing the nanoparticles in situ within the matrix.

\section{Envisaged applications}

As Niels Bohr once famously remarked, it is always difficult to make predictions, especially when they concern the future. Enthusiasts of nanotechnology assert that it will create revolutions in every branch of human endeavour. Due to the almost universal penetration of computing power into every branch of human activity, revolutions are likely by virtue of this indirect application alone. Whether the revolutions will be disruptive is difficult to predict. The world has been able to absorb internet and cellular telephone technology without so far significantly disrupting the fabric of civilization, and successive innovations are apparently absorbed with permanently increasing facility.

This section is mainly concerned with applications still being researched, but with sufficient weight of proof of principle for the nature of the application to be already clearly perceptible. The chief direct domains for which this appears to be the case are medicine and chemical synthesis and analysis. ${ }^{9}$

In the indirect realm, the main application of nanotechnology is in materials, particularly nanocomposites. The driving inspiration for their development is the notion that unique combinations of properties can be achieved by anastomosis at the nanometre scale. Many prototype materials are now being researched - there is particular interest in the aerospace industry in the use of ultralight and ultrastrong composites for structural members, and ultrahigh performance thermal barrier materials for turbine blade coatings — but the stringent safety

\footnotetext{
${ }^{9}$ These will spawn numerous indirect applications, of course, such as all the products made in chemical nanoreactors.
} 
requirements of most aerospace applications imply lengthy intervals of the order of a decade between laboratory demonstrations and large scale commercial use.

In the realm of ultraprecision machining, the coming generation of ultra high performance telescopes with aspherically machined mirrors having a surface roughness of a few nanometres are expected to enable astronomy based on visible wavelengths to forge ahead as never before, based on the construction of gigantic terrestrial telescopes, and all that implies in terms of new windows onto the universe, enabling fresh assessments of man and his relation to the cosmos.

\section{Medicine}

The current approach to pharmaceutical-based therapy, in which a drug is systemically absorbed by the whole body in order to affect a single localized organ, is totally antithetical to the concept of nanotechnology, according to which that organ, or diseased part of it, should be targeted with molecular precision. The pharmaceuticals in current use rely on slight differential selectivity of binding or uptake, and a dose sufficient to be effective against the diseased organ is likely to have significantly deleterious effects on the body as a whole when weak binding and uptake are summed over the entire rest of the body. The "smart medicine" or "magic bullet", targeting solely the organ of interest, has not yet been invented, but at least the conceptual basis of what is required is clear: the drug needs to be addressed to its target, much as a letter is addressed to an addressee. The present approach is akin to creating many copies of the letter and scattering them from an aeroplane over an entire city, where many people will pick them up and mostly discard them after cursory inspection.

An important area of imminent application is the implantable sensor for monitoring physiological parameters. Heartbeat, blood pressure and blood oxygenation are all routinely measured for patients in intensive care without the use of nanotechnology (although the powerful computing devices enabled by nanotechnology could be used for more sophisticated pattern recognition-based analysis of the data collected by those sensors); the next step in innovation will come through micro- or nano-sensors, based on minute electrodes or optical fibres, with which the concentrations of physiologically relevant molecules in the blood and other biofluids and tissues can be measured. The working principles of these sensors are well known, and some of them, notably the glucose sensor, are already in commercial use as off-line devices (i.e. samples are taken from the patient and applied to the sensor). Implanting these sensors in the body requires further advance in miniaturization, not only of the sensor itself but of its power supply and data transmission capability. If such sensors are successfully developed, they will be used not only in hospitals, but under normal circumstances of life whenever a person is considered to be, by himself or his physician, at risk of succumbing to some abnormality. For this approach to be really successful, the sensors need to be able to detect the incipient onset of disease, for which advances in medical knowledge unrelated to nanotechnology are needed, particularly the identification of novel biomarkers for different physiological states.

The third envisaged application of nanotechnology to medicine is in nanosurgery, a development of microsurgery. Although complicated operations still require to be carried out in 
the traditional way, the diminished invasiveness implied by microsurgical techniques makes them very attractive. The ultimate development in nanotechnology is considered to be quasiautonomous robots that can be released into the blood, through which they will travel to the site needing intervention, where they will carry out the required repair work. If they incorporate a camera - and miniature cameras able to travel through the digestive system while recording images already exist cand are commercially available - then the 'nanobot' could be controlled by external commands given by a surgeon. The possible algorithmic storage capacity of such devices - it is this that is likely to be the limiting feature, rather than miniaturization of surgical tools - is also being examined with the intention to establish whether a completely autonomous device is feasible, which could itself target the site requiring treatment and carry it out.

\section{Chemical synthesis and analysis}

Miniature reactors offer significant advantages over bulk reactions. In the terminology of nanotechnology, one has extreme control over every small packet of fluid moving through the reactor, allowing the outcome of the chemistry, which in nearly every system of practical interest is complicated enough to permit multiple outcomes, to be predicted with remarkable and unprecedented accuracy. Even before the era of nanotechnology, it was beginning to be realized that micromixing determined, to a primordial extent, the proportions of the different possible reaction products, and just how inefficient and difficult thorough mixing is in conventional large reaction vessels. Furthermore, in miniature reactors all parts of the fluid moving through the reactor are in close proximity to the surface, allowing surface catalysis to be exploited very efficiently.

Until now, effort has concentrated on applying top down methods to fabricate miniature reactors, which are mostly at the submillimetre scale at present, and in characterizing the reactions taking place within them from a physico-chemical viewpoint. Relatively little attention has been paid to evaluating the overall product cycle for microreactor-based manufacturing of large volumes of chemicals, in which issues of the integration of vast numbers of reactors operating in parallel have to be tackled. Clearly miniature reactor technology is especially wellsuited to making small batches of custom-synthesized chemicals.

Regarding chemical analysis ('lab-on-a-chip'), burgeoning research, and even a special journal exclusively devoted to the field, has resulted in the rapid accumulation of a large body of empirical data on the performance of a great variety of different miniature analytical systems. Significant advantages of the miniature systems - notably the need for only minute quantities of sample, very small quantities of reagents, low power consumption etc.- - have steadily driven down the size of systems for chemical analysis, and devices of varying degrees of miniaturization are already being exploited commercially.

\section{What are the main challenges facing nanotechnologists?}

In this final section, attention is drawn to those areas intrinsic to nanotechnology where there has been rather little progress to date. 
Self-assembly

How to design precursor molecules able to move along a definite pathway of connexions among themselves to yield a prespecified three dimensional structure is still an unsolved problem. A powerful inspiration for devising such molecules are natural proteins. The pathways they follow to achieve their final three dimensional structures with sophisticated functionalities have been intensively studied, and there is now some hope of making synthetic analogues that would self-connect according to the same principle, namely that of least action.

The nub of the problem is that there are too many possibilities of connexion for all to be tried. Thus, even if the final desired structure lay in a free energy minimum, on average too many fruitless sets of connexions would have to be sampled first, and the almost certain presence of local free energy minima could cause the search to become stuck in a system of connexions very different from the optimal one. In terms of the self-connecting of macromolecules, the principle is, more concretely, the principle of sequential minimization of entropy loss. ${ }^{10}$ In the true spirit of the principle of least action, as formalized by Boltzmann, the folding biomolecule follows a pathway by repeatedly updating its configuration so as to minimize the loss of entropy while maximizing the number of new contacts. This principle lies at the core of new, highly successful biopolymer-folding algorithms. The challenge now is to find algorithms to solve the inverse problem: when presented with a final structure, how should the precursor monomers be constructed?

\section{Molecular manufacturing}

There are three main problems facing the "assemblers" supposedly capable of putting together any kind of artefact. Firstly, they are of comparable size to the workpiece that they are trying to fashion. All conventional engineering devices for machining matter are much larger than the size of the object that they are designed to machine. This is to ensure that they are not deformed by the reaction of the workpiece on the tool, which, in accord with Newton's third law, is equal and opposite to the action of the tool on the workpiece. What will prevent the nano-assembler from being itself deformed and probably destroyed by the workpiece it is attempting to fashion? The argument applies with equal force to non-mechanical machine tools, e.g. the electric field at the tip of a scanning probe required to manipulate the atoms of a workpiece would have to be strong enough to cause the tip itself to disintegrate.

Secondly, interfacial forces make nanoscale objects very sticky. It is well known that the relative importance of different forces is scale-dependent: insects, but not humans, can transport unaided a burden twenty times their own weight, and walk on water. ${ }^{11}$ The mass of a body scales with the cube of its length; muscle strength with the square, but surface tension scales

\footnotetext{
${ }^{10} \mathrm{~A}$. Fernández and H. Cendra, "In vitro RNA folding: the principle of sequential minimization of entropy loss at work”. Biophys. Chem. 58 (1996) 335-339.

${ }^{11}$ Human ingenuity has of course enabled humans to perform much more remarkable feats with the aid of technology.
} 
linearly with characteristic length, hence it typically dominates not only in the nanometre régime, but often in the micrometre one too. Whereas this domination has often been regarded as a nuisance by the nanotechnologist, thwarting his attempts to position individual atoms or molecules with nanometre precision, it can also be exploited with advantage, to make miniature oscillators for example. ${ }^{12}$

Thirdly, it will be difficult for the nanoassemblers to store sufficient information for both their assembly tasks and self-replication. Ants and other social insects are often upheld as living proofs of principle for the molecular assembler concept (ignoring the fact that even the smallest such insects are many orders of magnitude bigger than the proposed assemblers). While it is perfectly true that the elaborate nests and other structures that they build seem to be based on a very small set of instructions modulated by information about the immediate environment (the so-called stigmergic construction principle), every nest is substantially different from another in detail — as indeed they must be in a natural environment, where every potential site also differs from another in detail - but at present it has not been worked out whether such principles could be used to construct the strictly standardized components, whose introduction was really crucial to the industrial revolution and the era of mass manufacturing. That is not to say that stigmergic principles cannot be exploited, only that the entire production cycle will need to be reexamined, and further advances in information storage capacity will doubtless be required.

\section{Nanoparticle safety}

Since nanoparticles are very small, they can penetrate into parts of the body that other forms of the same material cannot reach. A piece of aluminium is not considered to be toxiceven cooking utensils are routinely made from it, but aluminium nanoparticles ingested via the digestive system, or via skin penetration or the lungs, are likely to be highly toxic. Surprisingly little work has been done so far on the toxicology of nanoparticles and nanotubes, except where they are being used as medicines. This is partly due to familiarity with airborne micro- and nanoparticles, i.e. smoke. It is presumed that human beings are able to cope with smoke from garden bonfires, cigarettes, motor vehicle exhausts etc. This presumption needs to be examined more closely. While the chimneyless cottage common in Russia two hundred years ago has largely disappeared, the ubiquity of smoke even in modern living environments has somewhat inured society to its possible injurious effects. Nanotechnology itself offers the tools to investigate airborne particles - nanophotonics (integrated optics) for detecting and quantifying them, atomic force microscopy for auxiliary structural investigations, and so on. Medical science will of course be heavily involved in these investigations, which will impact not only human cell ultrastructure and effects on the immune system, but should also contribute to solving the riddle of allergy.

\footnotetext{
${ }^{12}$ B.C. Regan et al., " Surface-tension-driven nanoelectromechanical relaxation oscillator". Appl. Phys. Lett. 86 (2005) 123119.
} 


\section{Public acceptance}

Nanotechnologists have become concerned that their work may suffer the fate that has befallen genetically modified food (GMF) crops - public rejection. They should be reassured by considerable differences between the two realms. GMF is a highly focused technology impinging very directly on humans (where they are fed to animals, those animals are intended for human consumption). Nanotechnology, on the other hand, comprises an enormously diverse set of activities, many of which are already warmly embraced - some might say too warmly — by the overwhelming majority of the public. The mobile telephone is the best example. The relentless march towards miniaturization of almost all branches of technology means that the consequential rejection of nanotechnology will comprise rejection of almost every artefact associated with life at the beginning of the twenty first century. For example, even if nanocomposites have yet to significantly influence aircraft design, a modern airliner has hundreds of onboard computers all made using top down nanotechnology.

Public concerns about health hazards are often unpredictable, dictated more by fashion than by sober consderation of available facts. Indirect effects of nanotechnology - such as electromagnetic radiation from mobile telephones - might well be more deleterious than direct effects, such as inhalation of nanoparticles, especially if one discounts all those sources of nanoparticles, especially smokes, which have existed since time immemorial. What is needed is not complacency, but the serious investigation of all risks; there is still far too little data, and that lack often lends to their analyses the character of speculation.

\section{Summary and conclusions}

Nanotechnology, defined as both a technology for fabricating ultrasmall materials and devices, and a concept in which everything in the world is considered from the viewpoint of atomic or molecular building blocks, is already influencing a very broad range of human technological activity. What are the implications of nanotechnology for materials, devices and systems? The most immediate consequence of miniaturization of materials is the huge increase in surface area. Hence for any material whose performance depends on specific surface area, nanoparticles offer an immediate and automatic advantage. A further possible advantage is that the intrinsic properties of the material may be changed for the better when it is finely divided. This is particularly relevant for electronic and photonic devices, in which energy levels become discrete if the device is small enough, allowing size-dependent tuning of output energy, and other advantageous features (which are, however, lost in devices requiring arrays of particles if they are not uniform in size). Intriguingly, silicon's bandgap becomes direct rather than indirect if it is prepared in pieces smaller than $5 \mathrm{~nm}$, with significant implications for monolithic polyfunctional optoelectronic devices. Finally, material miniaturization potentially allows diverse properties to be efficiently combined in a single composite material, a combination that may be unattainable at the macro scale.

Probably nanoparticles represents the most widespread current form of nanomaterials. A huge variety of different types of particles are already available, ranging from simple ultraviolet 
absorbers used in sunscreens to highly sophisticated and polyfunctional particles used to control drug delivery, and in solar panels to harvest sunlight and convert it into electric current. Magnetic nanoparticles have already enormously enhanced the performance of memory and magnetic recording media. Carbon nanotubes show great promise as electron emitters, in which rôle they may soon replace cumbersome cathode ray tubes.

Most miniature devices that have been demonstrated are still, strictly speaking, micro devices. At the true nano scale, single electron devices are topics of intense research as the basis of ultraminiature electronic circuits for computing and other applications. Biological molecular motors are being intensively studied as a source of design inspiration for truly nanoscale motors. Nano-optics is the term now used to describe the active field of integrated optics, in which light is guided and controlled in structures whose dimension is considerably less than the wavelength of light. Typical optical waveguides usable with visible or infrared radiation are between 100 and 200 nanometers thick. The thin films deposited on top of optical waveguides in order to carry out control functions may be only ten nanometres thick. Nanodevices, particularly sensors for process control, have the advantage of being usable in highly confined spaces. They are also generally highly sensitive, and typically far less costly than larger devices. Furthermore, simply by being small, devices with different functions can be combined on a single chip, yielding novel polyfunctionality.

An overarching feature of nanotechnology is that it represents a viewpoint in which problems of the understanding of underlying mechanisms are solved at the nanometre scale. This often leads to unique insight into the operation of a process, enabling far better control to be devised, in turn leading to far higher quality of output. 